\title{
Sistema de Gestión de la Energía para una planta de conservas
}

\section{Energy Management System for a canning plant}

\author{
German Vásquez Araya* \\ *Universidad Técnica Nacional, Costa Rica. Contacto: gvasquez@utn.ac.cr \\ Referencia/ reference: \\ Vásquez, G. (2020). Sistema de Gestión de la Energía para una planta de conservas. Yulök Revista de Innovación Académica. \\ $4(1), 81-89$
}

\section{Resumen}

Este documento resume el desarrollo de un sistema de gestión de la energía con base en la norma ISO 50001:2018, para la planta principal de una empresa de conservas de productos del mar. El proceso se resume desde la etapa inicial en la que se detallan antecedentes, el problema, los fundamentos técnicos, entre otros aspectos. Posteriormente se describe y caracteriza un sistema de gestión de energía (SGEn) (ISO, 2018). y, finalmente, se desarrolla, una propuesta del sistema de gestión para la empresa bajo estudio, donde la implementación del SGEn pronostica obtener una reducción en la facturación anual de $7.48 \%$ que representa beneficios anuales de USD 175,137.09 para este caso.

Palabras clave: energía, ISO 50001, auditoría energética, balance energético, indicadores energéticos.

\section{Abstract}

This document summarizes the development of an energy management system based on the ISO 50001:2018 standard, for the main plant of a canned seafood company. The process is described from the initial stage where detail the background, the problem, the theory, and other aspects, then is describe and characterize what is an energy management system (EnMS) (ISO, 2018). and finally develops a proposal of the management system for the company under study. Where the implementation of the EnMS predicts a reduction in the annual turnover of $7.48 \%$ that represents annual benefits of USD 175,137.09 for this case.

Keywords: Energy, ISO 50001, Energy audit, Energy balance, Energy indicators. 


\section{Introducción}

Costa Rica ha establecido políticas energéticas para la sustentabilidad de los recursos naturales, así como medidas contra el cambio climático (MINAE, 2015) y en este documento se resume la implementación de una herramienta de gran valor como lo son los sistemas de gestión de la energía (SGEn) que han demostrado ser exitosos y logran mejoras significativas en el desempeño energético de las empresas, independientemente de sus características y particularidades. Por lo tanto, se busca un beneficio para las empresas, al reducir sus costos de producción, y un beneficio para la sociedad, al mitigar los efectos y los impactos del cambio climático en el desarrollo socioeconómico.

Adicionalmente, para una empresa que se encuentra en competencia, es muy importante ofrecer los mejores precios de sus productos con el fin de establecer ventajas competitivas que beneficien su crecimiento. Sin embargo, si no se cuenta con un conocimiento detallado de los costos energéticos y de sus indicadores, se pueden generar distorsiones, así como pérdidas económicas y de competitividad.

\section{Planteamiento del problema}

La incertidumbre se plantea como el problema principal en la gestión de la energía. Los indicadores energéticos muchas veces no están definidos, además, no se sabe cómo las variables afectan el desempeño energético de sus procesos. Esto limita la capacidad de reacción para la toma de decisiones, con el fin de mejorar en forma continua el desempeño energético (ISO, 2018).

Además, esta incertidumbre se puede resumir como el desconocimiento del desempeño energético de sus procesos, pues no se cuenta formalmente con un sistema de gestión de la energía, lo cual genera la desviación e impide tomar las mejores decisiones. Ante lo expuesto, se propone desarrollar un sistema de gestión de la energía con base en la norma ISO 50001 para los procesos más importantes, con el fin de evaluar continuamente su desempeño energético y determinar sus costos específicos con base en la norma ISO 50006.

\section{Qué es un Sistema de Gestión de la Energía (SGEn) ISO 50001}

De acuerdo con la organización internacional de estándares (ISO), el propósito de esta norma internacional es facilitar la implementación de los sistemas y procesos necesarios para mejorar el desempeño energético, la eficiencia energética y el uso y el consumo de la energía. Además, pretende reducir las emisiones de gases de efecto invernadero y de otros impactos ambientales relacionados, así como los costes de la energía, a través de una gestión sistemática. La norma es aplicable a organizaciones de todo tipo y tamaño, independientemente, de sus condiciones geográficas, culturales o sociales. Su implementación exitosa depende del compromiso de todos los niveles y funciones y, especialmente, de la Alta Dirección (ISO, 2018).

Al igual que otras normas, la 50001 se estructura en el ciclo de mejora continua: Planificar-Hacer-Verificar-Actuar (PHVA) y se puede detallar de la siguiente manera:

- Planificar: se centra en entender el comportamiento energético de la organización, para establecer los controles y objetivos necesarios que permitan mejorar el desempeño energético.

- Hacer: busca implantar procedimientos y procesos regulares, con el fin de controlar y mejorar el desempeño energético.

- Verificar: monitorear y medir procesos y productos con base en las políticas, objetivos y características clave de las operaciones y reportar los resultados.

- Actuar: tomar acciones para mejorar continuamente el desempeño energético, con base en los resultados.

Como se observa en la figura 1, cuando se inicia un sistema gestión de la energía se tienen muchas oportunidades para mejorar el desempeño energético con una inversión baja. Esto se debe a que muchos son cambios sencillos, como modificaciones de procedimientos de operación o mantenimiento, eliminación de malas prácticas, mejoras pequeñas de equipos, sin embargo, cuando el sistema va madurando las oportunidades de mejora se reducen, se vuelven más complejas y se requiere de una mayor inversión. 


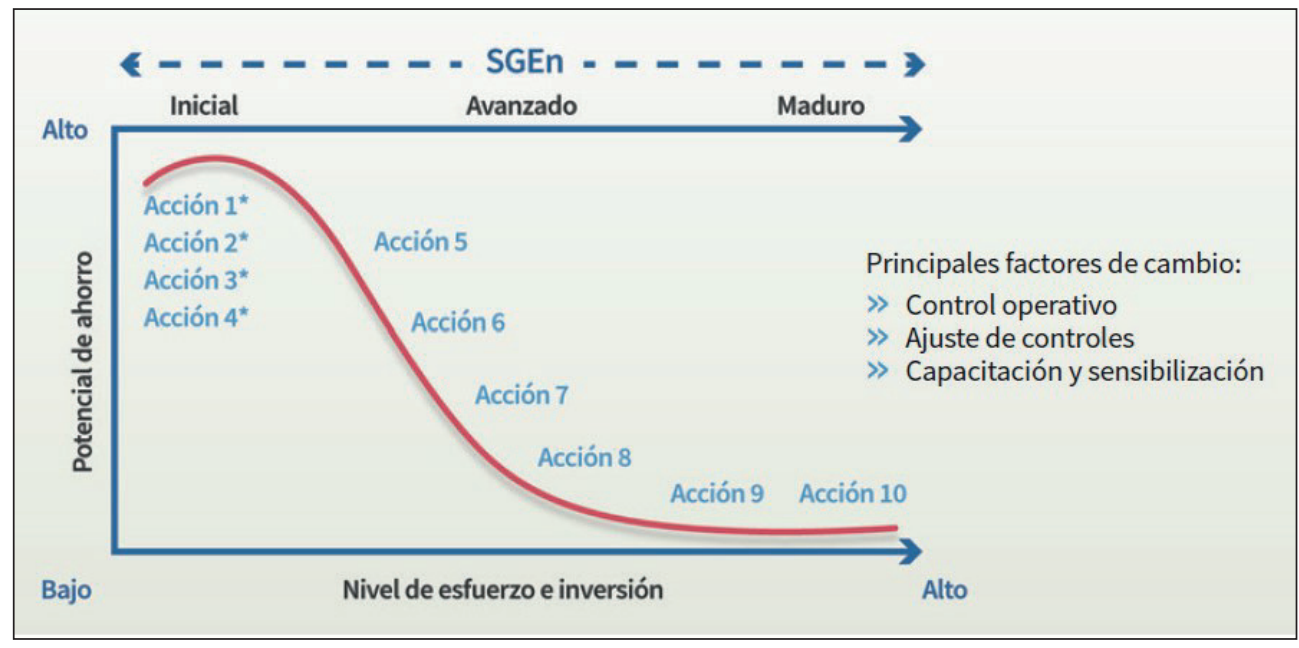

Figura 1. Evolución de un SGEn. Fuente: Rosas, 2018.

\section{Aplicación de una auditoría energética}

De acuerdo con la organización internacional de estándares, en la norma ISO 50002 (2018), una auditoría energética es un análisis sistemático del uso de la energía y su consumo, con el fin de identificar, cuantificar e informar sobre las oportunidades de mejora del desempeño energético.

$\mathrm{Al}$ igual que la Norma ISO 50001, la ISO 50002 no está asociada con un proceso específico, sino que se puede aplicar en cualquier empresa en la que se quiera determinar el desempeño energético, de una forma sistemática y estandarizada. Esto se puede hacer de manera periódica, para observar las mejoras y comparar los resultados.

De acuerdo con la norma, existen tres niveles de auditoría (I, II y III) y su aplicación dependerá de la complejidad del proceso, del grado del uso de la energía y de la inversión que requiera llevar a cabo la empresa. En la Norma ISO 50002 (2018) se detalla el alcance de cada uno de los tres niveles de auditoría.

\section{Diseño e implementación del Sistema de Gestión de la Energía}

En este punto se resume el diseño y desarrollo del Sistema de Gestión de la Energía para el caso en estudio se utilizó como guía el Manual para la Implementación de un Sistema de Gestión de la Energía en el contexto centroamericano, el cual fue desarrollado por varios países, en conjunto con la Comisión Nacional para el Uso Eficiente de la Energía (CONUEE) y con la asistencia técnica del Deutsche Gesellschaft für Internationale Zusammenarbeit (GIZ). Esta guía se ha utilizado en diversas empresas de Centroamérica con resultados muy satisfactorios.

Para el diseño del sistema de gestión de la energía se aplicaron los pasos que se observan en la tabla 1, adaptándolos al contexto y situación de la empresa y alineados con la norma ISO 50001 (2018).

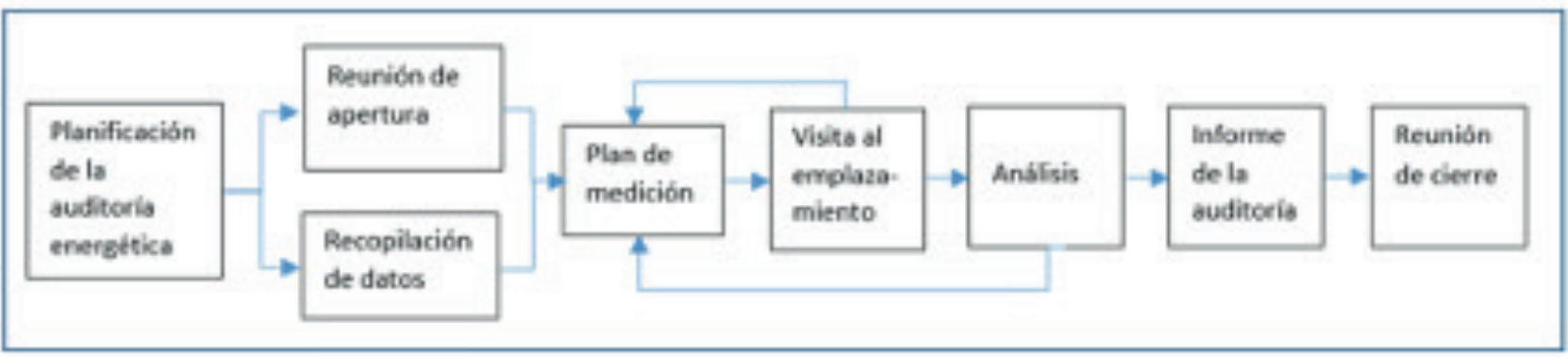

Figura 2. Diagrama de flujo del proceso de auditoría energética. Fuente: ISO 50002 (2018). 
Tabla 1. Pasos para el Diseño e Implementación de un SGEn

\begin{tabular}{|c|c|c|}
\hline $\begin{array}{c}\text { Ciclo de } \\
\text { mejora } \\
\text { continua }\end{array}$ & Etapa & Pasos \\
\hline \multirow[t]{2}{*}{ Inicio } & \multirow{2}{*}{$\begin{array}{l}\text { 1. Identificar el escenario ini- } \\
\text { cial }\end{array}$} & 1.1 Determinar el contexto de la organización \\
\hline & & $\begin{array}{l}\text { 1.2 Definir responsabilidades de la alta dirección, liderazgo y compro- } \\
\text { miso }\end{array}$ \\
\hline \multirow[t]{15}{*}{ Planear } & \multirow{4}{*}{$\begin{array}{l}\text { 2. Establecer el compromiso } \\
\text { con el SGEN }\end{array}$} & 2.1 Definir alcance y límites del SGEn \\
\hline & & 2.2 Definir un representante de la dirección \\
\hline & & 2.3 Establecer un equipo de gestión de la energía \\
\hline & & 2.4 Definir una política energética \\
\hline & \multirow{6}{*}{$\begin{array}{l}\text { 3. Evaluar el desempeño ener- } \\
\text { gético }\end{array}$} & 3.1 Identificar y evaluar requisitos legales y otros \\
\hline & & 3.2 Recopilar datos energéticos \\
\hline & & 3.3 Establecer usos significativos de energía \\
\hline & & 3.4 Definir la Línea de Base Energética e IDEN \\
\hline & & 3.5 Registrar oportunidades de mejora \\
\hline & & 3.6 Desarrollar un sistema de seguimiento \\
\hline & \multirow{3}{*}{$\begin{array}{l}\text { 4. Establecer objetivos y me- } \\
\text { tas }\end{array}$} & 4.1 Determinar el marco de trabajo \\
\hline & & 4.2 Estimar el potencial de mejora \\
\hline & & 4.3 Definir objetivos y metas \\
\hline & \multirow[t]{2}{*}{ 5. Crear planes de acción } & 5.1 Definir etapas y fines \\
\hline & & 5.2 Asignar funciones y destinar recursos \\
\hline \multirow[t]{6}{*}{ Hacer } & \multirow{6}{*}{$\begin{array}{l}\text { 6. Poner en práctica los planes } \\
\text { de acción }\end{array}$} & 6.1 Fortalecer competencias \\
\hline & & 6.2 Elaborar un plan de comunicación y sensibilización \\
\hline & & 6.3 Establecer la documentación del SGEn \\
\hline & & 6.4 Generar controles operacionales \\
\hline & & 6.5 Incorporar el desempeño energético en el proceso de diseño \\
\hline & & 6.6 Establecer criterios de compras \\
\hline \multirow[t]{3}{*}{ Verificar } & \multirow[t]{3}{*}{ 7. Evaluar el progreso } & 7.1 Dar seguimiento y control \\
\hline & & 7.2 Medir los resultados \\
\hline & & 7.3 Revisar los planes de acción y el SGEn \\
\hline \multirow[t]{3}{*}{ Actuar } & \multirow[t]{3}{*}{ 8. Reconocer Logros } & 8.1 Realizar revisiones por la dirección \\
\hline & & 8.2 Tomar decisiones para mejorar el SGEn \\
\hline & & 8.3 Evaluar la conformidad \\
\hline
\end{tabular}

Fuente: Rosas, 2018. 


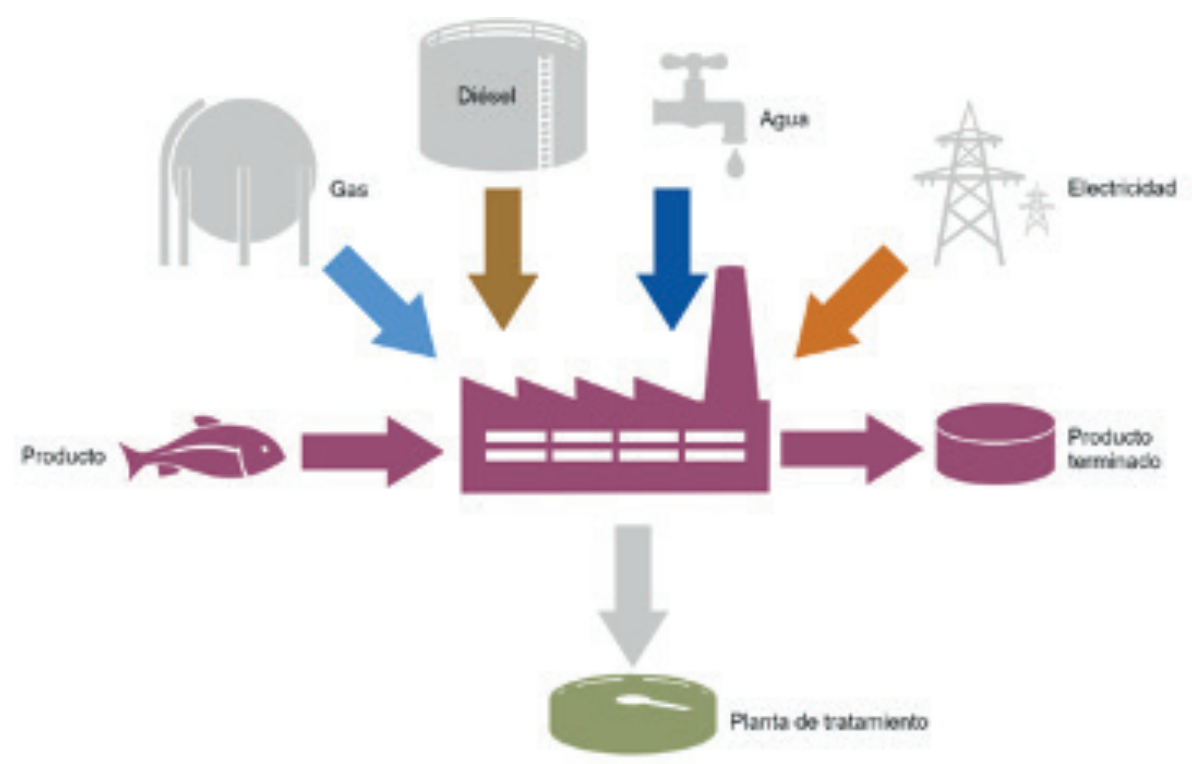

Figura 3. Diagrama de entradas y salidas del caso en estudio.Fuente: elaboración propia, 2019.

Con el fin de profundizar, se detallarán cada una de las etapas, con el objetivo de comprender como estas contribuyen en la implementación del SGEn.
Identificar el escenario inicial: con el fin de identificar la situación de la empresa, se requiere determinar el es- cenario inicial como punto de partida para el desarrollo del sistema de gestión de la energía. Para esto se propone que la empresa realice un análisis FODA (fortalezas, oportunidades, debilidades y amenazas) desde el punto de vista energético y con ello identificar los riesgos, para comprender las necesidades y expectativas de las partes

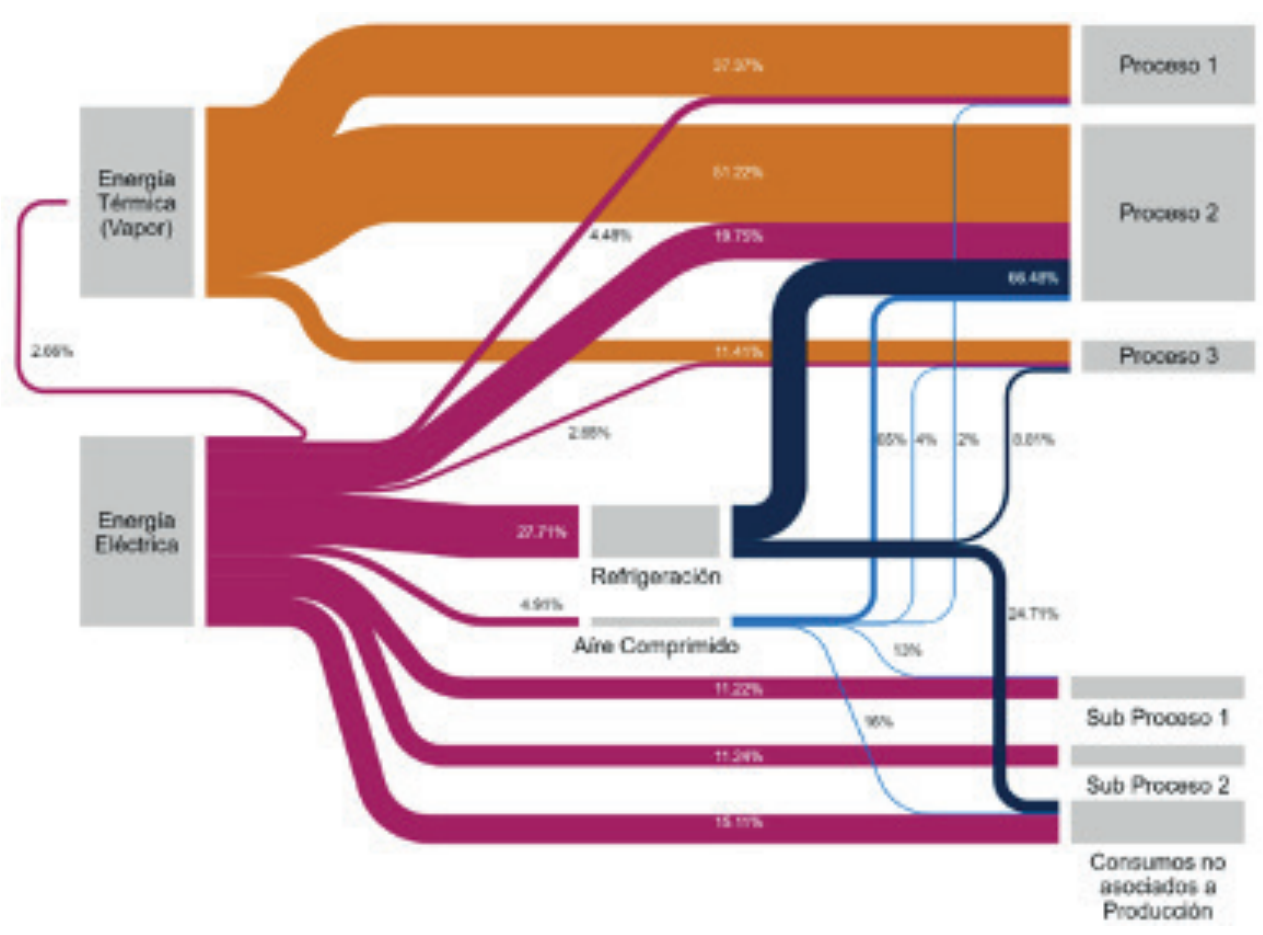

Figura 4. Balance energético de la empresa bajo estudio. Fuente: elaboración propia, 2019. 
Tabla 2. Línea base (LBEn) e indicadores de desempeño energético (IDEn)

\begin{tabular}{|l|l|l|l|}
\hline Fuente & IDEn & LBEn & IDEn Meta \\
\hline Energía eléctrica & $\mathrm{kWh} / \mathrm{t}$ & $446,62 \mathrm{kWh} / \mathrm{t}$ & $430 \mathrm{kWh} / \mathrm{t}$ \\
\hline Vapor & $\mathrm{Kg} / \mathrm{t}$ & $1557 \mathrm{~kg} / \mathrm{t}$ & $1500 \mathrm{~kg} / \mathrm{t}$ \\
\hline Aire comprimido & $\mathrm{CFM} / \mathrm{t}$ & $8,1 \mathrm{CFM} / \mathrm{t}$ & $8,0 \mathrm{CFM} / \mathrm{t}$ \\
\hline
\end{tabular}

Fuente: elaboración propia, 2019.

interesadas, el contexto de la organización y las responsabilidades de la Alta Dirección de la Empresa.

Establecer el compromiso con el SGEn: el compromiso de todas las partes interesadas del proceso es igual de importante que el compromiso de la Alta Dirección. En este punto se define el alcance y límites del SGEn, que en este caso es la planta de producción y los límites son las dos líneas de producción más representativas, además se identifican los integrantes del equipo de gestión de energía en el cual participan personas de las diversas áreas de la empresa y por último se establece una propuesta de la política energética de la empresa, la cual marcará el punto de partida y los objetivos energéticos de esta.

Evaluar el desempeño energético: para desarrollar este paso es indispensable conocer los procesos y las relaciones que existen entre ellos para determinar dónde y cómo se consume la energía. En este caso la empresa utiliza tres fuentes primarias de energía: LPG (vapor) y electricidad (diésel y externa). La figura 3 resume sus entradas y salidas.

Luego de analizar toda la información obtenida de la auditoría energética y de los instrumentos de medición se logra determinar el balance de energía de la empresa en estudio, el cual se resume en la figura 4.

También, de este punto nacen los indicadores y las líneas bases, los cuales de acuerdo con la norma ISO $50001 \mathrm{y}$ con el manual guía del SGEn (Rosas, 2018), una línea base energética (LBEn) es una referencia cuantitativa que proporciona la base de comparación del desempeño energético en un periodo definido (típicamente 1 año) y en el caso del indicador de desempeño energético (IDEn) este es un valor cuantitativo o una medida del desempeño energético y se define típicamente como una razón, (Ejemplo: energía/producción) aunque pueden ser elementos más complejos. Ambos se realizan para tener un punto de referencia para la mejora continua del desempeño energético de la empresa y por consiguiente de su competitividad.

Con base en los consumos y en la producción promedio de 1200 toneladas se definen tres indicadores globales: consumo especifico de producción global (CEPG), consumo térmico específico de producción global (CTEPG) y consumo eléctrico específico de producción global (CEEPG) y cuyos valores son los siguientes para este caso en estudio:

$$
\begin{aligned}
& \text { CEPG }=\frac{\text { Consumo total de Energia }}{\text { Producción }}=9973.64 \mathrm{MJ} / \mathrm{t} \quad \text { Ecuación } 1 \\
& \text { CTEPG }=\frac{\text { Consumo de Energia Térmica }}{\text { Producción }}=6662.53 \mathrm{MJ} / \mathrm{t} \quad \text { Ecuación } 2 \\
& \text { CEEPG }=\frac{\text { Consumo de Energia Electrica }}{\text { Producción }}=3308.33 \mathrm{MJ} / \mathrm{t} \quad \text { Ecuación } 3
\end{aligned}
$$

Con estos indicadores globales la empresa puede compararse con otras compañías de características similares con el fin de establecer el nivel de eficiencia global de sus procesos. Adicionalmente, se obtienen indicadores específicos (tabla 2) con el fin de dar seguimiento a la mejora continua en sus procesos internos.

De la auditoría nivel II realizada y del análisis de la información se encontraron varias oportunidades de mejora para este caso, las cuales se resumen en la tabla 3 y donde se observan las reducciones totales, el beneficio anual equivalente, conocido como BAUE y su periodo de recuperación. 
Tabla 3. Oportunidades de mejora para este caso en concreto

\begin{tabular}{|l|l|l|l|}
\hline \multicolumn{1}{|c|}{ Oportunidad de mejora } & \multicolumn{1}{|c|}{$\begin{array}{c}\text { Ahorros de ener- } \\
\text { gía anuales }\end{array}$} & \multicolumn{1}{|c|}{ BAUE } & \multicolumn{1}{|c|}{$\begin{array}{c}\text { Periodo de re- } \\
\text { cuperación }\end{array}$} \\
\hline 1. Cambio de la tarifa T-MT a T-MTb & N/A & USD 145,023.77 & 3 meses \\
\hline $\begin{array}{l}\text { 2. Cambio de motores por de alta eficien- } \\
\text { cia en los cocinadores. }\end{array}$ & N/A & USD 0 & Nunca \\
\hline $\begin{array}{l}\text { 3. Tuberías y equipos sin aislantes térmi- } \\
\text { cos. }\end{array}$ & $128091,6 \mathrm{~L}$ & USD 18,126.59 & 1 año \\
\hline $\begin{array}{l}\text { 4. Malas prácticas sobre las áreas con am- } \\
\text { bientes controlados y el cuarto de hielo. }\end{array}$ & $1477,15 \mathrm{kWh}$ & USD 184.64 & 3 meses \\
\hline $\begin{array}{l}\text { 5. Instalación de variadores de frecuencia } \\
\text { en los compresores de amoniaco. }\end{array}$ & $199.751,50 \mathrm{kWh}$ & USD 6,768.64 & 1,33 años \\
\hline $\begin{array}{l}\text { 6. Presión de ajuste de los compresores } \\
\text { mayor de lo que se requiere. }\end{array}$ & $30554.16 \mathrm{kWh}$ & USD 3,855.64 & Inmediato \\
\hline $\begin{array}{l}\text { 7. Excesos con aire comprimido en la lim- } \\
\text { pieza de latas }\end{array}$ & $11160 \mathrm{kWh}$ & USD 1,177.81 & 4 meses \\
\hline Reducciones totales & $242942,81 \mathrm{kWh}$ & USD 175,137.09 & \\
\hline
\end{tabular}

Fuente: elaboración propia, 2019.

Establecer objetivos y metas: una vez establecido el desempeño actual de la empresa (IDEn y LBEn) y la política energética, se deben definir los objetivos y metas energéticas, las cuales son indispensables para la mejora continua y el desarrollo del sistema de gestión de la energía. En este caso se definieron cuatro objetivos, los cuales se presentan a continuación.

1. Aumentar la eficiencia energética del proceso productivo reduciendo al menos $7 \%$ anual del consumo de energía.

2. Adquirir productos y servicios energéticamente eficientes en al menos $70 \%$ de las contrataciones ejecutadas anualmente.

3. Reducir las emisiones de efecto invernadero en al menos $5 \%$ anual por medio de la optimización de las fuentes de energía.

4. Desarrollar las competencias necesarias definidas en un plan de capacitaciones para el SGEn en todos los colaboradores de la empresa, en un periodo no mayor de tres años.

Crear planes de acción: definidos los objetivos y las metas, deben desarrollarse los planes de acción que logren cumplir con estos. Los planes se deben realizar con un grupo interdisciplinario que involucre las distintas áreas de la empresa y por medio de una lluvia de ideas generar un abanico de opciones, con el fin de escoger las que mejor se orienten en cumplir con los objetivos. Para ello, deben definirse las etapas y el fin de cada plan de acción, así como las actividades, responsables, recursos y fechas.

Poner en práctica los planes de acción: esta etapa consiste en implementar, dar seguimiento y evaluar los planes de acción que sean planteados por la organización con el fin de mejorar su desempeño energético, basado en la información del consumo, balance energético y usos de la energía, para ello se debe seguir una serie de pasos para que la implementación y cumplimiento de los planes de acción sean exitosos.

Evaluar el progreso: esta etapa se desarrolla una vez que se ha implementado y esté en curso el SGEn, consiste en evaluar todos los puntos desarrollados previamente y valorar el avance de cada uno de ellos con el fin de determinar la mejora del desempeño energético. El objetivo principal de la evaluación es establecer la efectividad de los planes de acción y los controles operacionales, y con ello establecer los cambios que se requieran con el fin de 
cumplir con las metas y en consecuencia con los objetivos energéticos.

Reconocer logros: este punto le corresponde a la Alta Dirección y con este actor se cierra el ciclo PHVA, específicamente en su componente Actuar. Le corresponde a la Alta Dirección la toma de decisiones y los reconocimientos de los responsables, de acuerdo con los resultados obtenidos de la evaluación, y para ello se requiere desarrollar tres pasos: revisiones periódicas por parte de la Alta Dirección, toma de decisiones para la mejora del SGEn y evaluar por medio de entes externos la conformidad de la aplicación de la norma ISO 50001 (auditorías externas).

El sistema de gestión de energía propuesto pretende ser sencillo y práctico con el fin de facilitar su implementación y desarrollo, en este se aplicaron todos los pasos necesarios para iniciar el proceso de implementación y de mejora continua, el cual debe validarse y mejorarse en cada ciclo PHVA.

\section{Beneficios del SGEn propuesto}

En este punto se resumen los resultados esperados y se muestran los beneficios económicos y ambientales que se tendrían al desarrollar e implementar el SGEn en este caso en particular.

Estos resultados funcionan como promotores del sistema de gestión de la energía, el cual con la mejora continua traería beneficios año con año, lo que al final representa una mejora sostenida en la competitividad de la empresa.

\section{Reducción de la huella de carbono}

Un beneficio adicional de las oportunidades de mejora es que la mayoría de los casos se reduce el consumo de las fuentes de energía y esto a su vez representa una reducción en el CO2 equivalente de la empresa. Para el caso de las oportunidades de mejora desarrolladas se obtiene una reducción anual de 242942,81 kWh en energía eléctrica y 128 091,6 L de GLP. Lo que representa una reducción de 224,86 toneladas de $\mathrm{CO} 2$ equivalente $(2,76 \%)$.

Finalmente, como resultado de esta propuesta se presentan las conclusiones, así como una serie de recomendaciones, las cuales se deben implementar en el futuro cercano para este caso en estudio.

\section{Conclusiones}

Se determinó el desempeño energético de los procesos de la planta de producción por medio de la auditoría energética nivel II, basada en la ISO 50002 (2014). Lo que representa la situación energética actual de la empresa y con ello se identificaron seis oportunidades de mejora.

Se obtuvo el balance de energía de la empresa (figura 4), lo cual representa un insumo importante para la toma de decisiones, determinación de las prioridades de inversión, su estructura de costos y el conocimiento de la forma en que se utiliza la energía en la empresa. Se determinó los principales usos de energía térmica y en el caso de la energía eléctrica la facilidad de refrigeración representa el $27,7 \%$, con base en el consumo total de la empresa.

Se obtuvieron indicadores de desempeño energéticos y las líneas base según la norma ISO 50006 (2014), para la energía eléctrica, el vapor y el aire comprimido con los

Tabla 4. Análisis de los costos actuales y beneficios económicos esperados del SGEn

\begin{tabular}{|c|c|c|}
\hline \multirow{2}{*}{ Rubro } & \multicolumn{2}{|c|}{ Costos } \\
\hline \multirow{2}{*}{ Electricidad } & Monto en colones & Monto en dólares \\
\hline GLP & $\mathbb{~} 710.827 .337,00$ & USD $1,184,712.23$ \\
\hline Total & $\mathbb{C} 694.034 .071,20$ & USD $1,156,723.45$ \\
\hline Ahorro & $\mathbb{C} 1.404 .861 .408,20$ & USD $2,341,435.68$ \\
\hline \% Ahorro & $\mathbb{1} 105.082 .254,00$ & USD $175,137.09$ \\
\hline
\end{tabular}

Fuente: elaboración propia, 2019. 
siguientes valores $446,62 \mathrm{kWh} / \mathrm{t}, 1557 \mathrm{~kg} / \mathrm{t}$ y $8,1 \mathrm{CFM} / \mathrm{t}$, respectivamente, los cuales representan de manera efectiva la situación energética de la planta de producción para el periodo 2017-2018.

Se determinó por medio de análisis de costos y beneficios que con las oportunidades de mejora y la implementación del SGEn se logra obtener una reducción en la factura anual de 7,48 \%, lo cual supera la meta de $7 \%$ y representa beneficios anuales de USD 175,137.09. En estos beneficios se está considerando las inversiones.

Se calcula que de implementarse las oportunidades de mejora se lograría una reducción anual de 242 942,81 kWh en energía eléctrica y 128 091,6 L de GLP, lo que representa una reducción de 224,86 toneladas de CO2 equivalente $(2,76 \%)$.

Del análisis de los datos se determinó que el factor estático de energía eléctrica de la planta representa el 55,66 $\%$ del total de la empresa. A esta situación se le debe dar seguimiento porque representa un costo muy elevado. A su vez este factor estático significa que para bajos niveles de producción los indicadores energéticos se verán afectados en mayor proporción hacia el alza (negativo). Estos consumos están asociados principalmente al sistema de refrigeración, el cual siempre debe estar encendido, sin importar la cantidad de producto y las cargas administrativas (no asociadas a la producción).

\section{Recomendaciones}

Contratar una evaluación exhaustiva de la eficiencia energética del sistema de refrigeración para encontrar oportunidades de mejora, debido a que es un sistema complejo, representa un consumo importante $(27,7 \%)$ y requiere de un análisis detallado de su operación. De esta evaluación implementar como IDEn al coeficiente de desempeño (COP) que representa la razón de la capacidad total de refrigeración obtenida entre la potencia aplicada al fluido.

El consumo de GLP representa un costo significativo, por lo que se recomienda contratar una revisión de la eficiencia de las calderas, con el fin de garantizar que se tiene la mejor eficiencia posible de ambos equipos y dar seguimiento semestral.

Realizar un levantamiento de todos los motores instalados y monitorear su operación anual, en caso de que sea mayor a $4000 \mathrm{~h}$ analizar su sustitución por motores de alta eficiencia.
Desarrollar un programa de mantenimiento predictivo con ultrasonido y termografía con periocidad trimestral, que incluya el tema de pérdidas térmicas tanto en vapor como refrigeración y que se realicen informes de las correcciones y estado actual.

\section{Referencias}

Agencia Chilena de Eficiencia Energética. (2012). Guía de Implementación de Sistema de Gestión de la Energía basada en la ISO 50001. Segunda edición.

ISO. (2011). 50001, Sistemas de Gestión de la Energía. https://www.iso.org/obp/ui/\#iso:std:iso:50001:ed-1:v1:es

ISO. (2018). 50001, Sistemas de Gestión de la Energia. https://www.iso.org/obp/ui/\#iso:std:iso:50001:ed-1:v1:es

ISO. (2018). 50002, Auditorías energéticas- Requisitos con orientación para su uso. https://www.iso.org/ obp/ui/\#iso:std:iso:50001:ed-1:v1:es

ISO. (2014). 50006, Sistemas de gestión energética-Medición del rendimiento energético, utilizando líneas de base de energía y los indicadores de desempeño energético - Principios generales y guía. https:// www.iso.org/obp/ui/\#iso:std:iso:50001:ed-1:v1:es

Ministerio de Ambiente y Energía (Minae). (2015). VII plan nacional de energía 2015-2030. San José, Costa Rica. Programa de las Naciones Unidas para el Desarrollo PNUD.

Rosas-Moya, R. (2018). Manual para la implementación de un sistema de gestión de la energía en el contexto centroamericano.

Instituto Meteorológico Nacional. (2018). Factores de emisión de gases de efecto invernadero. 